\title{
THE STRUCTURE OF THIN ZIRCONIA FILMS OBTAINED BY SELF-ASSEMBLED MONOLAYER MEDIATED DEPOSITION: TEM AND HREM STUDY
}

\author{
V.V. Roddatis, D.S. Su*, E. Beckmann, F.C. Jentoft, U. Braun, J. Kröhnert and R. Schlögl
}

Fritz-Haber-Institut der Max-Planck-Gesellschaft, Abteilung Anorganische Chemie, Faradayweg 4-6, D-14195, Berlin, Germany.

\begin{abstract}
Transmission electron microscopy (TEM), electron energy-loss spectroscopy (EELS) and energy dispersive X-ray analysis (EDX) have been performed on thin zirconia films produced by means of self-assembled monolayer (SAM) mediated deposition from aqueous zirconium sulphate dispersion at $50^{\circ} \mathrm{C}$. As-grown films were found to be amorphous. Electron beam irradiation can induce the crystallization of as-grown amorphous zirconia films to tetragonal polycrystalline $\mathrm{ZrO}_{2}$ films. EELS revealed changes in the oxygen K-edge peak caused by the beam-induced structural transition of the amorphous phase to tetragonal $\mathrm{ZrO}_{2}$.
\end{abstract}

Keywords: Self-assembled monolayer; Zirconia; TEM; Structure

*corresponding author, e-mail: dangsheng@FHI-berlin.mpg.de

Fritz-Haber-Institut der Max-Planck-Gesellschaft, Abteilung Anorganische Chemie, Faradayweg 4-6, D-14195, Berlin, Germany. 


\section{Introduction}

Zirconia (zirconium dioxide: $\mathrm{ZrO}_{2}$ ) is widely used in a number of different applications, some of which require the preparation of a thin layer of zirconia. The properties of such thin zirconia films depend on their structure and phase composition. Zirconia appears in three polymorphous phases: monoclinic $\left(\mathrm{m}-\mathrm{ZrO}_{2}\right)$ up to $1100^{\circ} \mathrm{C}$ [1], tetragonal $\left(\mathrm{t}-\mathrm{ZrO}_{2}\right)$ up to $2285^{\circ} \mathrm{C}$ [2], and cubic $\left(\mathrm{c}-\mathrm{ZrO}_{2}\right)$ above $2285^{\circ} \mathrm{C}$ [3]. The high temperature cubic phase is unstable at room temperature, but can be stabilized by doping $\mathrm{ZrO}_{2}$ with other oxides, such as $\mathrm{Y}_{2} \mathrm{O}_{3}, \mathrm{MgO}$ and $\mathrm{CeO}_{2}$ [4]. The tetragonal phase can also be stabilized at room temperature by incorporation of cationic impurities [5].

Besides the various traditional techniques for the preparation of zirconia layers (such as sputtering, vapor deposition, plasma spraying, sol-gel processes and so on), a new technique has been developed that uses a self-assembled monolayer (SAM) to grow thin films of metal oxides or their hydroxide precursors [6]. Specifically, bifunctional surfactant molecules are attached to a substrate through their surface active head groups, and a SAM is created spontaneously on the substrate through van der Waals interaction between the surfactant's long hydrocarbon chains. The SAM-coated wafer is immersed into a deposition medium containing a dissolved metal salt and a film (for simplicity further referred to as "zirconia film") is formed on the SAM-covered substrate after a certain deposition time.

In the present work, we investigate microstructure and electron beam sensitivity of zirconia films deposited with the SAM technique by means of TEM, EDX and EELS. 


\section{Experiment}

Single-crystal (100) silicon wafers (CZ, $750 \mu \mathrm{m}$ thick, p-type, polished on both sides) were cleaned using lab tissues and solvents, i.e. sequentially chloroform, acetone, and ethanol. The specimens were then subsequently treated in $\mathrm{NH}_{3} / \mathrm{H}_{2} \mathrm{O}_{2}(50: 50)$ at $70^{\circ} \mathrm{C}, \mathrm{HCl} / \mathrm{H}_{2} \mathrm{O}_{2}(50: 50)$ at $70^{\circ} \mathrm{C}$, and a $30: 70$ mixture of $30 \% \mathrm{H}_{2} \mathrm{O}_{2}$ and concentrated $\mathrm{H}_{2} \mathrm{SO}_{4}$ ('piranha'-solution) at $80^{\circ} \mathrm{C}$ (approx. 25 min per treatment). In order to form the SAM, the wafers were immersed in a solution of $50 \mu \mathrm{l}$ ethanethioic acid S-[16-(trichlorosilyl)hexadecyl]ester in $5 \mathrm{ml}$ bicyclohexyl for $5 \mathrm{~h}$ under $\mathrm{Ar}$ atmosphere (glove bag). Oxidation of the hydrophobic thioacetate group to the desired hydrophilic sulfonic acid terminating group was achieved by immersion into a saturated solution of $\mathrm{KHSO}_{5} * \mathrm{KHSO}_{4} * \mathrm{~K}_{2} \mathrm{SO}_{4}$ for 3-5 hours. Afterwards, the wafers were rinsed with distilled water and immediately transferred into the deposition medium, a $4 \mathrm{mM}$ solution of zirconium (IV) sulfate tetrahydrate (97\%, Alfa, Karlsruhe, Germany) in $0.4 \mathrm{~N}$ hydrochloric acid. Glass vials with the wafer and $50 \mathrm{ml}$ of deposition solution were placed into a $50^{\circ} \mathrm{C}$ water bath. Depositions were performed for 3, 12, 48 and 96 $\mathrm{h}$, and the deposition medium remained clear under these conditions. The specimens were rinsed with distilled water and blown dry with Ar under ambient conditions.

The samples were prepared for electron microscopy by conventional procedures including polishing, followed by ion milling. The specimens were investigated in a Philips CM200 electron microscope with a field emission gun operated at $200 \mathrm{kV}$, additionally featuring a Gatan imaging filter GIF100 and an energy dispersive X-ray spectrometer. Low temperature TEM investigations were performed in a Philips CM20 FEG (SOPHIE) [7] operated at 160 $\mathrm{kV}$. This microscope is equipped with a special cryogenic superconducting objective lens allowing the observation of specimens at liquid helium temperature. HRTEM was performed 
on samples with different deposition times using a JEOL 4000FX electron microscope operated at $400 \mathrm{kV}$.

\section{Results}

\subsection{The structure of as-grown films}

High-resolution images of films after $3 \mathrm{~h}$ growth revealed a thin dark band along the surface of the Si substrate indicating the formation of the first monolayers of the zirconia film. The dark band, i.e. the film, increased in thickness with deposition time in an almost linear fashion. The averaged values of the film thickness estimated from high-resolution crosssection images are plotted as a function of deposition time in Fig. 1. Uniform thickness over areas as large as $100 \mathrm{~nm}^{2}$ was observed for $48 \mathrm{~h}$ and $96 \mathrm{~h}$ samples, and the roughness was about 3-5 $\mathrm{nm}$. We did not observe saturation (growth rate decreases to zero with prolonged deposition time) as it was reported in [8] for deposition at $70^{\circ} \mathrm{C}$. The medium is stable at $50^{\circ} \mathrm{C}$ though, while at $70^{\circ} \mathrm{C}$ homogeneous nucleation and precipitation set in after about 30 min and the deposition medium is presumably depleted of zirconium species.

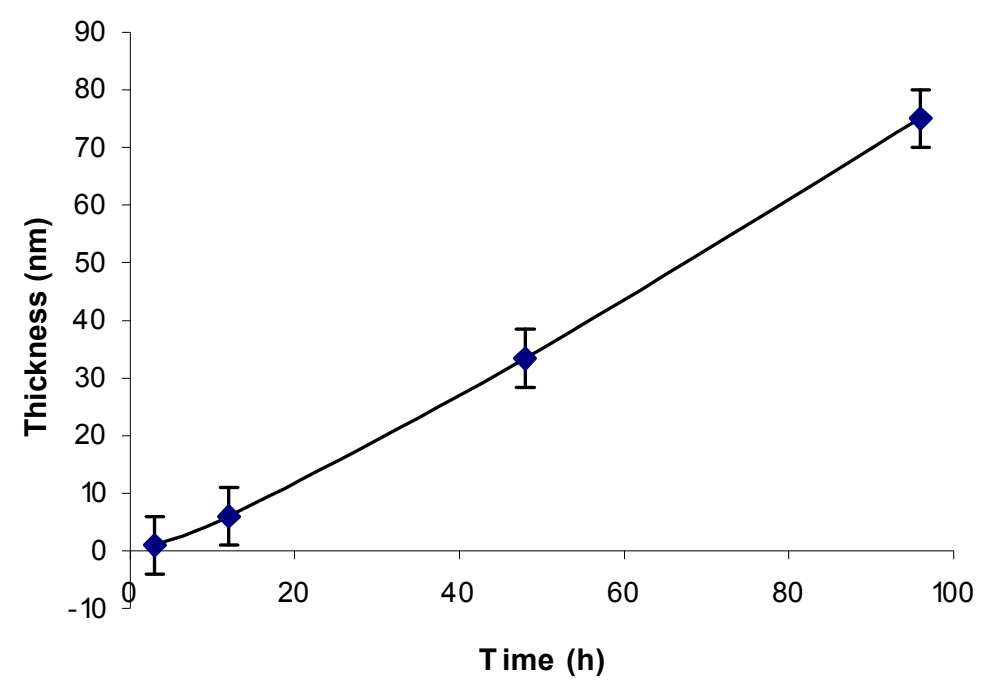

Fig.1. Plot of film thickness versus time. 
A typical HRTEM image of an as-grown film (48h) is shown in Fig. 2a. The film exhibits an amorphous nature (an enlarged image is inserted). No grains or crystalline structures could be found.
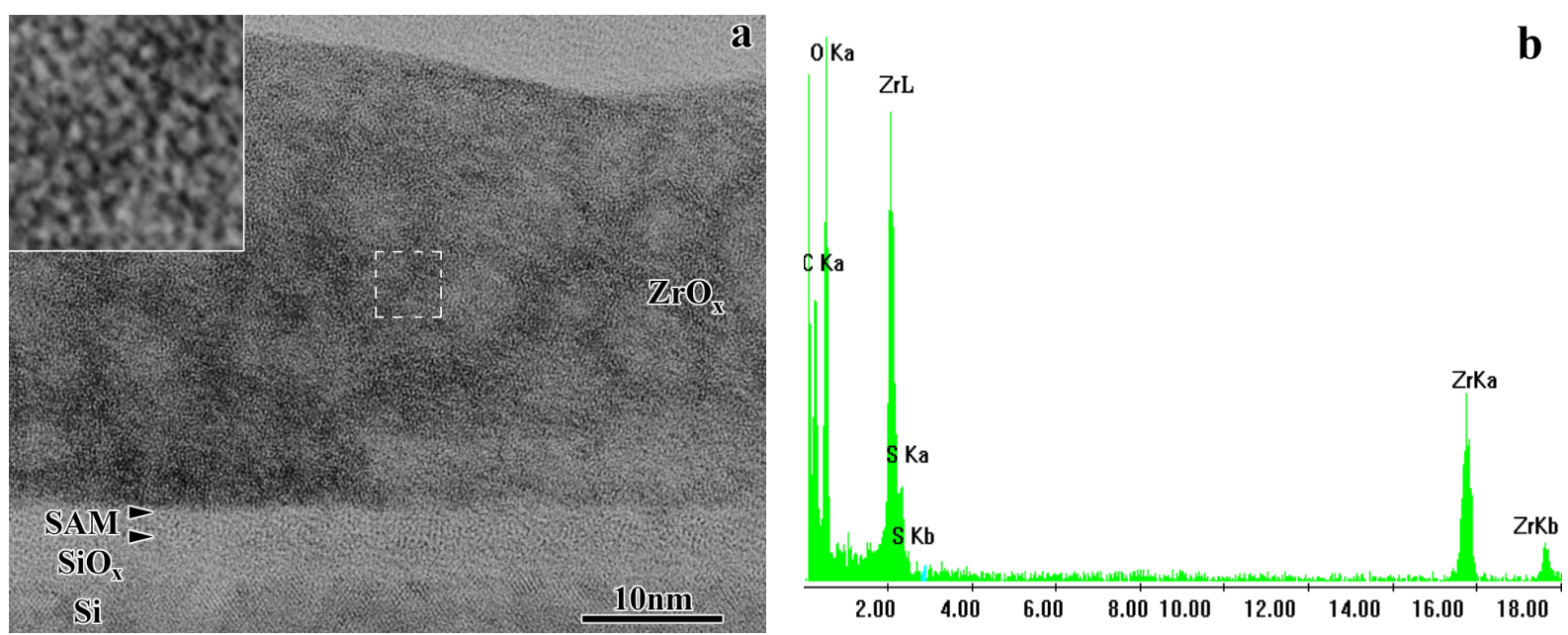

Fig.2. (a) HRTEM image of as-grown film (48h); (b) Corresponding EDX spectrum of asgrown film.

The calculated power diffraction spectrum (not shown here) shows a diffuse ring typical for amorphous structure. The amorphous state of as-grown films is not induced by ion milling in a Gatan PIPS because samples prepared by ion milling in a Gatan 600 at liquid $\mathrm{N}_{2}$ temperature were also amorphous. The images show areas of different contrast in the films, light round areas $(\sim 7 \mathrm{~nm}$ diameter $)$ are apparent in a dark matrix. The same features were also observed in [8] but their origin is still unclear. The layer between the zirconia film and the silicon substrate consists of two bands exhibiting slightly different contrast. These bands are assigned to the presence of an $\mathrm{SiO}_{\mathrm{x}}$ layer formed by oxidation of the $\mathrm{Si}$ substrate and to the presence of SAM [9]. A typical EDX spectrum of a film is shown in Fig. 2b. Besides zirconium and oxygen, sulfur was detected (up to $5 \mathrm{wt} \%$ ). The carbon signal is ascribed to the SAM which consists of long chain hydrocarbon molecules. 


\subsection{Irradiation induced changes}

The as-grown zirconia films are very sensitive to the electron beam. During investigations in the electron microscope it was observed that electron beam irradiation can induce crystallization of as-grown zirconia films. Two selected HRTEM images from a series obtained from the same area of the sample ( $48 \mathrm{~h}$, shown in Fig. $2 \mathrm{a})$ at various times of exposure to irradiation using the SOPHIE microscope are shown in Fig. 3. For instance, after irradiation with a current density of $0.5 \mathrm{~A} / \mathrm{cm}^{2}$ at $4.2 \mathrm{~K}$ for 1 minute (Fig. 3a) small crystallites were found in the film. The number of grains as well as their size increased with time, and after 10 min Moiré fringes became visible.
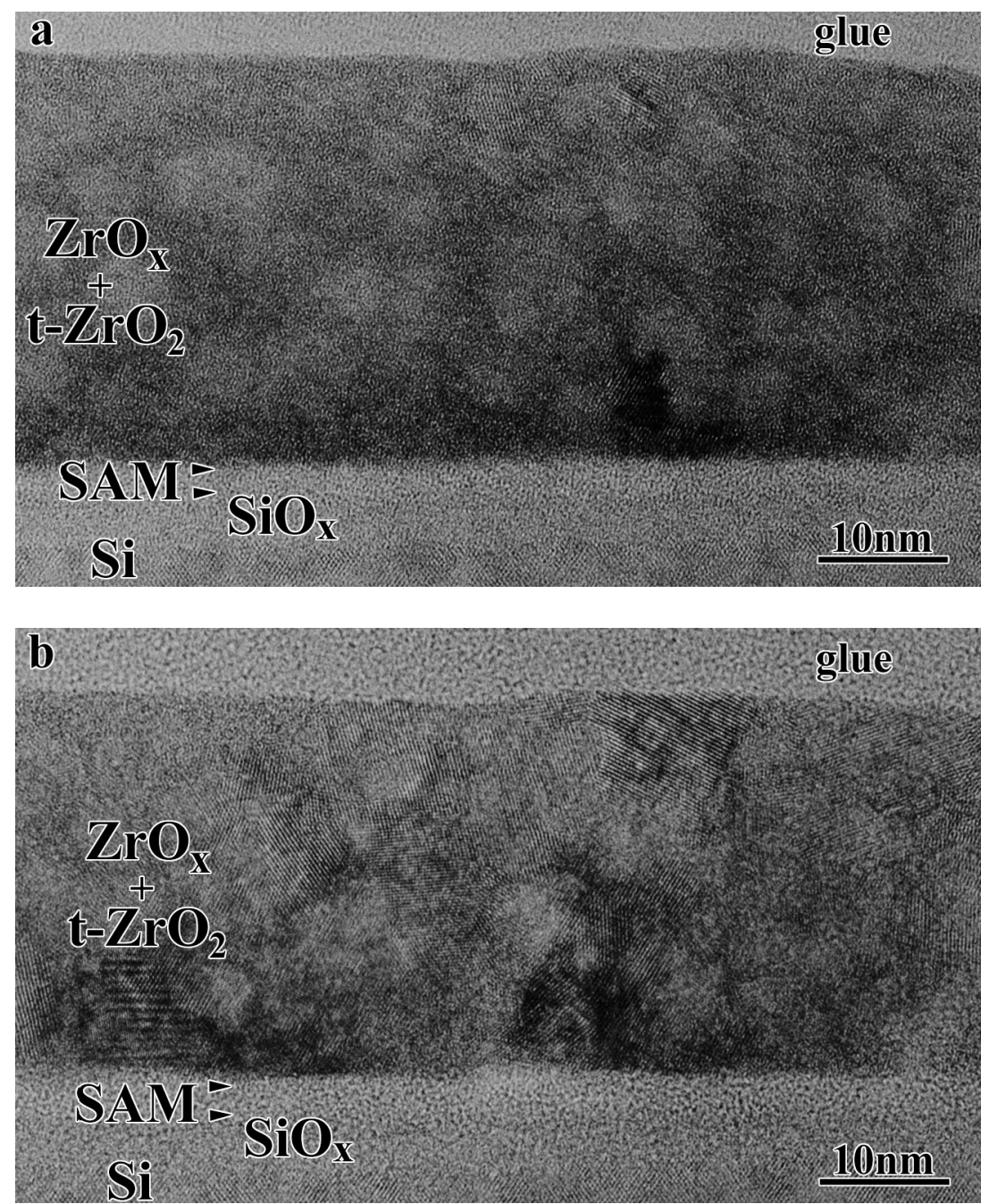

Fig. 3. HRTEM images of zirconia film (48 h). (a) after $1 \mathrm{~min}$, (b) after 20 min of electron irradiation at liquid helium temperature. Irradiation with current density of $0.5 \mathrm{~A} / \mathrm{cm}^{2}$. 
After 20 minutes the investigated area of the film consisted of grains about $10 \mathrm{~nm}$ in size (Fig. 3b). Using the Si lattice fringes as reference, FFT analysis confirmed that these grains belong to the tetragonal phase of $\mathrm{ZrO}_{2}$. The film thickness decreased by $6-10 \%$ during irradiation. Without cooling the sample to $4.2 \mathrm{~K}$ the crystallization proceeded much more rapidly (within less than $1 \mathrm{~min}$ ), i.e. the rate of crystallization depends on the ambient temperature. Radiation induced crystallization was also observed when using the JEOL 4000FX TEM at $400 \mathrm{kV}$, but slowly than in case of $200 \mathrm{kV}$ irradiation. Since with the increase of incident energy a cross-section for elastic scattering increases while a crosssection for inelastice scattering decreases, the observed crystallization suggests a process determined by inelastic scattering of electrons in the film.

\subsection{EELS measurements.}

In order to analyse the coordination of atoms in the zirconia films, oxygen K-edge spectra (1s transition) were obtained using EELS. Powders of tetragonal and monoclinic $\mathrm{ZrO}_{2}$ phases were used to record reference spectra. Energy loss spectra from an as-grown zirconia film were measured at the very beginning and after 5 minutes of electron irradiation. All spectra, after background subtraction and removal of multiple scattering are shown in Fig. 4. Loss of oxygen during electron irradiation could not be detected. The overall appearance of the spectra of the tetragonal reference, the monoclinic reference, and the film after electron irradiation is similar. Nevertheless, the spectra exhibit differences especially at energies above $545 \mathrm{eV}$. Subtraction of the spectrum of the crystallized film from the reference spectra shows the spectrum to resemble rather the spectrum of the tetragonal than the monoclinic phase. Small differences could be related to sulphur and amorphous components that are still present in the irradiated films. 
It is worth noting that the spectrum of the film at the beginning of electron irradiation does not show a pronounced peak at $\approx 534 \mathrm{eV}$ and does not have a peak at $544 \mathrm{eV}$. The maximum of this spectrum shifts, in comparison with other three spectra, to higher energy loss $(\approx 2.5$ $\mathrm{eV})$. Therefore, we can conclude that the as-grown films do not contain oxygen atoms with the same coordination as this one in tetragonal or monoclinic phases. The difference between the spectra 1 and other three spectra above $550 \mathrm{eV}$ can be due to the absence of long-range order in the as-grown films; for a detailed explanation spectra simulation is required.

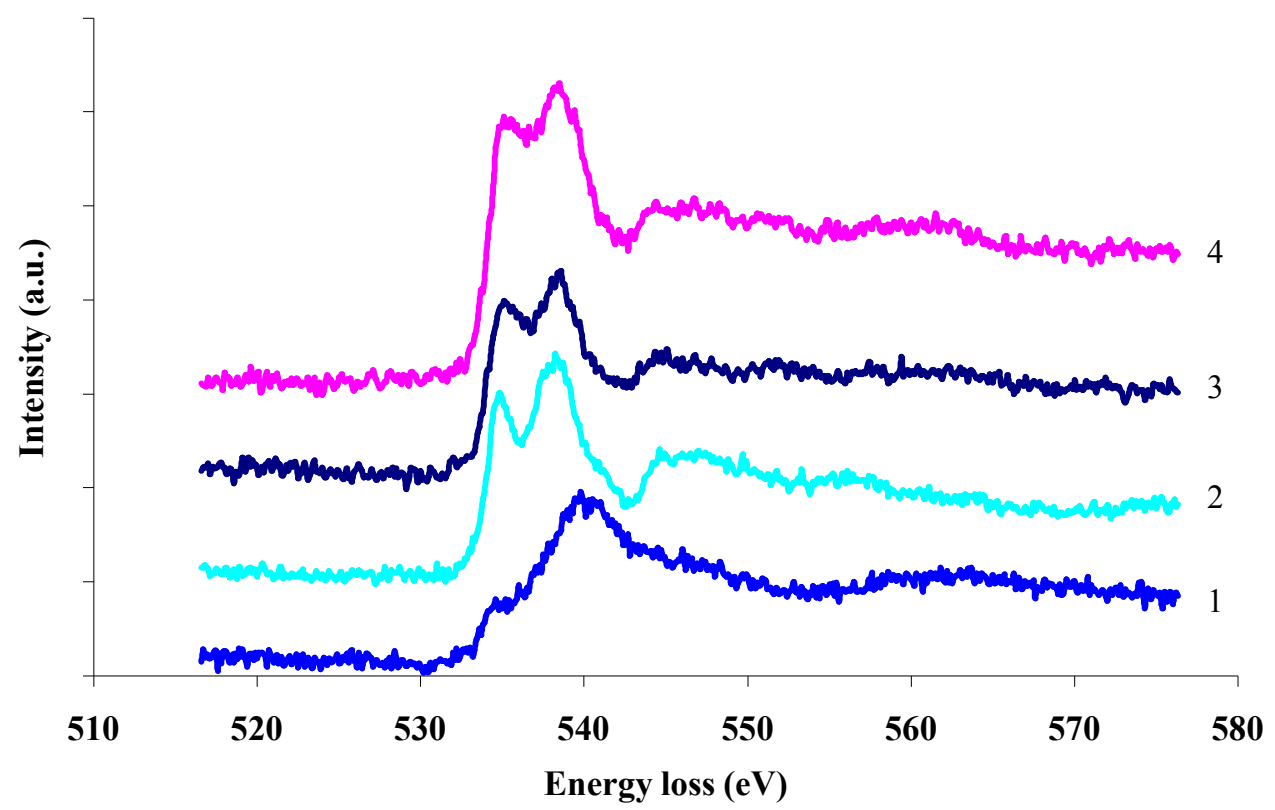

Fig. 4. Energy loss spectra: (1) film before irradiation with the electron beam; (2) after 5 min of irradiation; (3) tetragonal $\mathrm{ZrO}_{2}$, and (4) monoclinic $\mathrm{ZrO}_{2}$.

\section{Summary}

The thickness of zirconia films obtained at $50^{\circ} \mathrm{C}$ by self-assembled monolayer mediated deposition was found to increase linearly with time over about $96 \mathrm{~h}$, giving evidence of stable deposition conditions throughout the experiment. Film thickness can thus be tuned easily. As- 
grown zirconia films were found to be without long range structure. Crystallization of asgrown (non-annealed) films was observed under electron beam irradiation even when the sample was kept at liquid helium temperature. In the irradiated area, 5 to $12 \mathrm{~nm}$ sized grains exhibiting the structure of the tetragonal modification of $\mathrm{ZrO}_{2}$ appeared. This crystallization process was observed irrespective of the acceleration voltage (electron energy) but was influenced by the sample ambient temperature. Energy loss spectra revealed different oxygen coordination in as-grown film than in tetragonal or monoclinic phases, changed during irradiation too. Sulfur was detected by EDX analysis in as-grown and irradiated films, suggesting the presence of other species besides zirconium oxides.

\section{Acknowledgements}

VVR is indebted to the Max-Planck-Society for his fellowship at the Fritz-Haber-Institut.

\section{References}

[1].M.R. De Guire, H. Shin, R. Collins, M. Agarwal, C.N. Sukenik, A.H. Heuer. Proceedings of SPIE - the International Society for Optical Engineering, vol. 2686, (1996), 88-99.

[2].R.N. Patil and E.C. Subbarao, J. Appl. Crystallogr., 2, (1969), 281.

[3].P. Aldebert and J.-P. Traverse, J. Am. Ceram. Soc., 68, (1985), 34.

[4].E. Ryshkewitch and D.W. Richerson, Oxide ceramics, Physical Chemistry and Technology, (General ceramics, Haskell, NJ, 1985).

[5].P.D.L. Mercera, J.G. van Ommen, E.B.M. Doesburg, A.J. Burggraaf and J.R.H. Ross, Appl. Catal. 78, (1991), 79. 
[6].B.C. Bunker, P.C. Rieke, B.J. Tarasevich, A.A. Campbell, G.E. Fryxell, G.L. Graff, L. Song, J. Liu, J.W. Virden, and G.L. McVay, Science 264, (1994), 48.

[7].F. Zemlin, E. Beckmann, K.D. van der Mast, Ultramicroscopy 63, 3-4, (1996), 227.

[8].M. Agarwal, M.R. De Guire, A.H. Heuer, J. Am. Ceram. Soc., 80, (1997), 2967-81.

[9].A.D. Polli, T. Wagner, A. Fischer, G. Weinberg, F.C. Jentoft, R. Schlögl and M. Rühle, Thin Solid Films, 379, (2000), 122-127. 